\title{
El trabajo remoto y la gestión de las emociones en tiempos de la COVID-19: Una mirada de estudiantes de maestría desde el rol de trabajadores, Lima-Perú (2020)
}

\author{
Justina URIBE KaJAT \\ Kelly Myriam JiméneZ de Aliaga ${ }^{2}$ \\ José Gerardo Vargas Dávila ${ }^{3}$ \\ Daniel Enrique Rey de Castro Hidalgo ${ }^{4}$ \\ Miguel Ángel Bashualdo Pérez ${ }^{5}$ \\ Luis Alberto Geraldo CAMpos ${ }^{6}$
}

ReCibido: 15/03/2021 Aceptado: 24/04/2021 Publicado: 26/07/2021

\begin{abstract}
RESUMEN
El presente estudio tiene como objetivo principal determinar la relación entre el trabajo remoto y la gestión de las emociones. Asimismo, pretende determinar la relación entre las dimensiones jornada de trabajo, soporte laboral y tecnológico y bienestar social, y la variable gestión de las emociones. Se utilizó una metodología basada en un enfoque cuantitativo de alcance descriptivo-correlacional y de corte transversal sobre una muestra de 148 estudiantes de maestría de la Facultad de Administración de la Universidad Nacional Mayor de San Marcos. Los datos se obtuvieron a través de encuestas con escala tipo Likert. Los métodos de análisis fueron descriptivo y correlacional. Los resultados indican que las variables trabajo remoto y gestión de las emociones se correlacionan de forma positiva y moderada (0.465), por lo que se concluye que el trabajo remoto y todas sus dimensiones se relacionan con la gestión de las emociones.
\end{abstract}

Palabras clave: trabajo remoto; gestión de las emociones; estudiantes de maestría; trabajadores; COVID-19.

\section{INTRODUCCIÓN}

La crisis que hoy experimenta la humanidad, producto de la emergencia sanitaria de la COVID-19, es considerada por la Organización Mundial de la Salud como una pandemia (OMS, 2020) que ha cambiado la rutina y cotidianidad de las personas. El mundo del trabajo como lo concebimos ha cambiado; en Europa, según la Organización Internacional del Trabajo (OIT), "EI aumento más significativo del teletrabajo tuvo lugar en los países más afectados por el virus y en los que el teletrabajo estaba bien desarrollado antes de la pandemia" (2020, p. 3); en el Perú, ya se tenía una legislación, aunque incipiente, sobre el teletrabajo.

Hasta la primera semana de marzo de 2020 se tenía información sobre el comportamiento de la COVID-19 en Europa, principalmente en Italia, Francia y España, en los cuales la pandemia ya estaba dejando secuelas mortales. En su cronología sobre la actuación de la COVID-19 correspondiente al 11 de marzo de 2020, la OMS informó que estaba "Profundamente preocupada por los alarmantes niveles de propagación de la enfermedad

1 Doctora en Administración y doctora en Educación por la Universidad Nacional Federico Villarreal (Lima, Perú). Actualmente es docente en la misma universidad. (Lima, Perú). ORCID: https://orcid.org/0000-0002-2691-3073

E-mail: juribe@unmsm.edu.pe

2 Doctora en Enfermería por la Universidad Federal de Río de Janeiro (Río de Janeiro, Brasil). Docente investigadora en la Universidad Cesar Vallejo. (Lima, Perú). ORCID: https://orcid.org/0000-0002-8959-265X

E-mail: kmjimenez@ucv.edu.pe

3 Magíster en Gestión Pública. Actualmente es docente de la Universidad Nacional Mayor de San Marcos. (Lima, Perú).

ORCID: https://orcid.org/0000-0002-4914-8108

E-mail: jvargasda@unmsm.edu.pe

4 Magíster en Gestión Empresarial. Actualmente, es consultor independiente.(Lima, Perú). ORCID: https://orcid.org/0000-0002-0610-2150

Autor de correspondencia: danielreydecastro@gmail.com

5 Licenciado en Administración por la Universidad Nacional Federico Villareal. Especialista en Gestión de Capital Humano. Actualmente es consultor del Ministerio Público del Perú. (Lima, Perú).

ORCID: https://orcid.org/0000-0001-6456-8592

E-mail: miguelj1528@gmail.com

6 Magíster en Dirección y Administración de Empresas. Actualmente es docente en la Universidad Peruana Unión. (Lima, Perú).

ORCID: https://orcid.org/0000-0002-8366-689X

E-mail: luis.geraldo@upeu.edu.pe 
y por su gravedad, y por los niveles también alarmantes de inacción" y determinó en su evaluación que "la COVID-19 puede caracterizarse como una pandemia" (párr. 20). A partir de esa fecha, la mayoría de los gobiernos del mundo adoptaron medidas para evitar la propagación del virus en sus países.

En el Perú, ante la pandemia de la COVID-19, el 11 de marzo de 2020 se declaró en Emergencia Sanitaria a todo el territorio nacional por un período de 90 días calendario mediante un decreto supremo (D. S. No. 008-2020-SA, 2020). Asimismo, con esta norma legal se adoptaron medidas de prevención y control de alcance a todos los sectores de la actividad pública y privada.

Por esta razón, el Gobierno del Perú mediante el Decreto de Urgencia No. 026-2020, entre otras medidas, estableció el trabajo remoto con el propósito de salvaguardar la seguridad y salud de los trabajadores; por consiguiente, se estableció una forma diferente de trabajar a la que estábamos acostumbrados. La alarmante situación, desde ese momento oficial en el país, generó en las empresas incertidumbre y la necesidad de replantear sus procesos en pleno aislamiento social. En este sentido, Juárez (2021) señaló que la COVID-19 “tomó a las organizaciones por sorpresa, en pleno proceso de implementación de su transformación digital, mientras que otras aún no tenían al trabajo remoto como una opción" (párr. 2). Este contexto se convirtió, en definitiva, en una realidad irrebatible.

Se debe precisar que uno de los antecedentes del teletrabajo y el trabajo remoto es el Convenio 177 de la OIT (1966) sobre trabajo a domicilio, el cual estableció que es aquel que se realiza en el propio domicilio o en cualquier otro lugar distinto del que ofrece el empleador; en este contexto, los trabajadores reciben una remuneración, prestan un servicio o elaboran un producto de acuerdo a los requerimientos del empleador que es una persona jurídica. Esta norma supranacional insta a los Estados a la protección de todos los trabajadores, es decir, a abarcar todos los derechos laborales. Como se advierte, en ninguno de los extremos del citado convenio se menciona el uso de tecnologías de la información y comunicación. A la fecha, este convenio solo ha sido ratificado por diez países entre los cuales no se encuentra el Perú.

En este marco, Eurofound y OIT (2019) en su informe "Trabajar en cualquier momento y en cualquier lugar: consecuencias en el ámbito laboral” presentan los resultados de una investigación efectuada en 15 países observados ${ }^{7}$; al referirse al teletrabajo/TIC-trabajo móvil (T/TICM) ${ }^{8}$, señalan que las TIC han causado una revolución en el trabajo y en la vida de las personas y han hecho posible la conexión en tiempo real con la familia, así como con los jefes inmediatos y compañeros de trabajo. Asimismo, consideran que el aspecto negativo de esta nueva forma de trabajo es "la intrusión del trabajo remunerado en los espacios y tiempos reservados para la vida personal" (p. 8); sin embargo, advierten también, que los avances tecnológicos son favorables para los empleados porque impulsan la "flexibilidad espacial y temporal para conciliar las demandas laborales con sus responsabilidades familiares y personales" (p.62). Del mismo modo, los resultados del T/TICM son positivos para los empresarios, en mérito que les permitieron conseguir un mejor rendimiento y la optimización de la productividad.

En efecto, las TIC han transformado la actividad empresarial, así como la vida de las personas. En países desarrollados, ya se tenía un avance significativo en el sistema de teletrabajo y otras modalidades a distancia; sin embargo, en países como el Perú, con las TIC en desarrollo inicial, se han tenido que poner en marcha sistemas de trabajo en función de su propia realidad. En este contexto, el gobierno peruano optó por establecer el trabajo remoto.

En la actualidad, se utilizan los términos teletrabajo y trabajo remoto indistintamente, como si tuvieran el mismo concepto y aplicación; sin embargo, son distintos. En la legislación peruana, el teletrabajo se regula mediante la Ley No. 30036, D. S. No. 017-2015-TR, que básicamente establece que se requiere la aceptación del trabajador para su puesta en práctica, las labores son subordinadas y no requieren de asistencia física. Esta normativa es de aplicación en los sectores público y privado al tratarse de una modalidad en la que el vínculo laboral se mantiene y se lleva cabo mediante el uso de las TIC en sus diferentes formatos $y$, a través de ella, el empleador ejerce el control y la supervisión laboral. El suministro de herramientas informáticas y otros métodos que coadyuven al desempeño de la función corroboran el carácter de subordinación.

En cambio, el trabajo remoto es regulado por el Decreto de Urgencia No. 026-2020 y no es de libre elección por el trabajador. Las circunstancias de la pandemia obligaron a su puesta en práctica para evitar contagios en la población; es por esta razón, que el servicio que presta el trabajador en el trabajo

8 T/TICM es definido en el estudio como el uso de las TIC como, incluidos ordenadores, móviles, laptops, para prestar servicios en relación de dependencia, pero fuera de las instalaciones de la empresa empleadora. laborales 
remoto lo realiza en su domicilio o en el lugar de aislamiento domiciliario que es conocido por el empleador. Con este propósito, se adoptaron medidas orientadoras para los empleadores con el fin de no afectar, entre otros aspectos, la relación laboral y las remuneraciones, y mantenerlos informados sobre los cuidados de salud y seguridad que debían observar. Por otra parte, para los trabajadores se dictaron también normas sobre seguridad y confidencialidad de la información, el cumplimiento de las medidas de seguridad y salud en el lugar de aislamiento, y disponibilidad durante la jornada de trabajo. De manera similar que en el teletrabajo, se hace uso de las TIC u otros medios para el cumplimiento de las funciones en la medida que la naturaleza de estas lo permitan.

Así pues, el trabajo remoto es un novísimo sistema de trabajo que surgió como consecuencia de la pandemia de la COVID-19 y, a la fecha, se desconocen los efectos o la relación entre el trabajo remoto y la gestión de las emociones de los trabajadores, aun cuando existen algunos estudios e investigaciones relacionados al teletrabajo y su efecto sobre las emociones.

Weller (2020), al referirse al trabajo a distancia y teletrabajo en el marco de la pandemia, señala que la digitalización ha ayudado en el ámbito laboral y también ha contribuido a la "conciliación entre el trabajo y la vida familiar, la descongestión del tráfico urbano" (p. 12); afirma además que se espera el crecimiento de trabajos apoyados en plataformas digitalizadas a nivel global. Weller (2020) y López (2020) coinciden en que el trabajo a distancia en épocas de la COVID-19 ayuda a evitar la expansión de la pandemia en los centros laborales y, por ende, en el hogar.

En efecto, se considera que en tanto la pandemia continúe, y probablemente después de ella, la presencialidad será necesaria en casos puntuales como los centros de producción, la industria del agro, las minas, entre otras actividades económicas; sin embargo, el trabajo a distancia será una modalidad cuya opción de permanencia en el tiempo no se descarta.

En su artículo, Peralta et al. (2020) analizan el impacto del teletrabajo en las empresas como resultado, principalmente, al avance de las TIC; afirman que este tiene una incidencia en el área laboral y ha obligado a las empresas a adoptar nuevas modalidades de trabajo como el teletrabajo. En este sentido, en una de sus conclusiones señalan que el teletrabajo "rompe los esquemas tradicionales y genera en el teletrabajador mayor confianza en la organización y sentido de pertenencia, lo que persigue y consecuentemente espera una mayor productividad y eficiencia en los procesos administrativos" (p. 9), lo que se puede lograr porque esta modalidad forma parte de la flexibilidad laboral.

Johnson et al. (2020) desarrollan una serie de reflexiones sobre las emociones frente a la pandemia de la COVID-19, y concluyen que existe "la necesidad de diseñar estrategias para disminuir la incertidumbre con el objetivo de mejorar la salud de la población, considerando las desigualdades sociales y de género existentes" (p. 2455). Estas reflexiones deberían ser aplicadas a todos los trabajadores, sea cual fuere la actividad que desarrollen.

La pandemia de la COVID-19 tiene preocupados no solo a los gobiernos, sino también a los organismos internacionales como la OMS y la OIT. Ambas han emitido normas para que los gobiernos las adopten de acuerdo a sus circunstancias. En efecto, la vulnerabilidad no es igual en todos los países, las respuestas se dan en función a los avances científicos y tecnológicos.

En la publicación del suplemento Día1 del diario El Comercio del Perú sobre el trabajo remoto, Chávez (2021) presenta resultados positivos obtenidos por FutureLab sobre el manejo de las emociones en este periodo de emergencia sanitaria. Uno de los aspectos importantes que abordados fue la necesidad de que las empresas busquen la preservación de la salud de la familia de los colaboradores. Esta posición concuerda también con las conclusiones de un artículo publicado recientemente por Estrategias y Negocios (2021).

La presente investigación aborda dos variables: el trabajo remoto y la gestión de las emociones. El trabajo remoto fue abordado teniendo en cuenta el derecho del trabajador al respeto de la jornada de trabajo y a la desconexión digital (D. U. No. 1272020) y analizado desde tres dimensiones: jornada de trabajo, soporte laboral y tecnológico, y bienestar social.

La jornada de trabajo se conceptualiza como "el tiempo que cada trabajador dedica a la ejecución del trabajo para el que ha sido contratado. Esta jornada tiene un límite establecido por la Ley que es de 8 horas diarias o 48 horas semanales, como máximo" (Ministerio de Trabajo y Promoción del Empleo, 2019 , p. 10); por otra parte, el soporte laboral tecnológico debe entenderse como las actividades de apoyo a los trabajadores que gestionan las entidades en momentos de pandemia y se evidencian en directrices para el óptimo desarrollo de sus funcio- 
nes, la transferencia de información, los programas tecnológicos, los equipos y otras herramientas que faciliten el trabajo remoto. Finalmente, el bienestar social comprende actividades destinadas a generar un adecuado ambiente de trabajo y una mejor calidad de vida de los servidores (MINJUS-SERVIR, 2016).

El análisis de la inteligencia emocional obliga a los investigadores a evocar los aportes de Gardner (2014), quien considera que las inteligencias son capacidades o dominios intelectuales que despliegan habilidades específicas para fines específicos, lo que da lugar a la teoría de las inteligencias múltiples. Es a partir de esta, y en particular de los tipos de inteligencia intrapersonal e interpersonal, que Salovey y Mayer (1990) proponen por primera vez el concepto de inteligencia emocional con énfasis en los procesos cognitivos de evaluación y expresión emocional, así como la regulación emocional.

Sobre las emociones en una situación crítica de pandemia, Ekman (2013) señala que tienen un valioso significado para la vida, debido a que permiten sentir placer y exteriorizar alegría, así como también permiten experimentar otros tipos de sentimientos como ira, odio o celos; todos ellos, generados por estímulos externos. Las emociones, tal como lo señalaron Cotrufo y Ureña (2018), tienen una función sumamente importante en contextos cambiantes de crisis e incertidumbre como los que se viven en estos tiempos, y esta es la de adaptación, tal como lo sustentó Charles Darwin en 1872 en una publicación que denominó "La expresión de las emociones en el hombre y en los animales".

En estas circunstancias, es importante el interés que se pone en el impacto de la pandemia sobre las emociones, como señalan Johnson, Saletti-Cuesta y Tumas (2020) cuando afirman que es fundamental el énfasis en la salud mental "no solo para mejorar la salud sino también para evitar otros problemas sociales, tales como la estigmatización de personas, la falta de adherencia a medidas de prevención, y el duelo frente a la pérdida de seres queridos" (p. 2448). En esta línea coinciden con Manucci (2017) en que el desarrollo de la capacidad emocional de las personas es impostergable, puesto que las personas que realizan trabajo remoto no son ajenas al impacto emocional de la pandemia.

Para comprender la gestión emocional, se debe partir por conocer lo que son las emociones. Goleman (1996) afirmó que "Todas las emociones son, en esencia, impulsos que nos llevan a actuar, programas de reacción automática con los que nos ha do- tado la evolución [...] sugiriendo, de ese modo, que en toda emoción hay implícita una tendencia a la acción" (p.24), y que la emoción "se refiere a un sentimiento y a los pensamientos, los estados biológicos, los estados psicológicos y el tipo de tendencias a la acción que lo caracterizan" (p.331). Goleman señaló además que existen algunas emociones primarias: ira, tristeza, temor, placer, amor, sorpresa, disgusto y vergüenza. Cotrufo y Ureña (2018) aportan desde la neurociencia y consideran que las emociones "son un conjunto de cambios que se dan a nivel fisiológicos, cognitivos, subjetivos y motores" (p.18) y surgen de una percepción consciente o inconsciente a partir de las circunstancias y de la realidad concreta (estímulos) en la que interactúa una persona.

De acuerdo a Goleman (1996), la autoconciencia está relacionada con la capacidad de conocer las propias emociones y tener la habilidad para identificar un sentimiento mientras ocurre; la autorregulación consiste en la capacidad de las personas para canalizar adecuadamente los sentimientos, partiendo de la conciencia del propio ser; y la motivación está relacionada con la capacidad de estructurar adecuadamente las emociones para alcanzar objetivos mejor enfocados y priorizados. A su vez, la empatía se refiere a la capacidad para reconocer y comprender las emociones de los demás, lo que, en cierta manera, permite la adaptación de las personas a contextos de crisis como la que se vive por la pandemia del coronavirus. Finalmente, las habilidades sociales están relacionadas con la capacidad para gestionar adecuadamente las relaciones con los demás. En consecuencia, tal como se señaló en Gálvez (2017), la gestión emocional es la habilidad de las personas para regular las emociones y adaptarse a los cambios que se puedan presentar en el entorno de interacción de las personas.

Por consiguiente, la investigación busca responder la siguiente pregunta: ¿cuál es la relación que existe entre el trabajo remoto y la gestión de las emociones? Además, tiene como objetivo general determinar la relación que existe entre el trabajo remoto y la gestión de las emociones de los estudiantes de la maestría de Administración con mención en Dirección de Recursos Humanos, en su condición de trabajadores del sector público o privado. Asimismo, se pretendió determinar la relación entre las dimensiones jornada de trabajo, soporte laboral tecnológico y bienestar social, y la variable gestión de las emociones.

En definitiva, la situación de pandemia por la que atraviesa el mundo ha generado nuevas formas de convivencia comúnmente llamadas "nueva normalidad" donde el distanciamiento físico es una de las 
normas fundamentales. Este contexto obligó a la mayoría de gobiernos a instaurar el trabajo remoto, una modalidad de trabajo a distancia impuesta e implementada sin un marco legal ni soporte tecnológico suficientemente sólido en muchos países, entre ellos el Perú. En este sentido, teniendo en cuenta al capital humano como el elemento intangible más importante en las organizaciones, resulta significativo el estudio del impacto del trabajo remoto en los trabajadores, desde las dimensiones fundamentales para el buen desempeño laboral. Los aportes de la presente investigación profundizan y exploran un tema nuevo y poco estudiado debido a la rápida y sorpresiva propagación de la pandemia de COVID-19 y sus consecuencias en el planeta. En el Perú, el trabajo remoto es una experiencia muy reciente $y$, como tal, presenta nuevos retos a las organizaciones y entidades tanto públicas como privadas en lo que respecta a la implementación de estrategias de gestión efectivas que garanticen, por un lado, la productividad y el óptimo desempeño de los trabajadores, y por otro, el bienestar y salud emocional de los mismos. De la misma forma, esta investigación es un aporte para el conocimiento, fundamentalmente sobre la percepción y comportamiento de los trabajadores frente a situaciones críticas e inesperadas que exijan la introducción de nuevas modalidades de trabajo. Finalmente, esta investigación puede servir como base para futuros estudios académicos.

\section{MATERIALES Y MÉTODOS}

El estudio tiene un enfoque descriptivo, pues como Rivas (2017) señala "describe al sujeto de investigación, sin hacer juicios de valor sobre él pero analizando las descripciones y buscando asociaciones entre ellas" (p.128). Es correlacional porque, a decir del mismo autor, "buscan medir el grado en que están asociadas dos o más variables" (p.132). Es de corte transversal porque se centra en conseguir datos de un determinado hecho en un momento determinado (Arbaiza, 2016).

La muestra estuvo conformada por 148 participantes de la maestría en Administración con mención en Dirección de Recursos Humanos, periodo académico 2020, de la Unidad de Posgrado de la Facultad de Ciencias Administrativas de la Universidad Nacional Mayor de San Marcos, Lima Perú.

Los instrumentos aplicados a los participantes fueron dos. El primero corresponde a la variable trabajo remoto e incluye 26 ítems distribuidos en tres dimensiones: jornada de trabajo, soporte laboral y tecnológico, y bienestar social; el segundo fue diseñado para medir la variable gestión de las emociones e incluye 20 ítems distribuidos en cinco dimensiones: autoconciencia, autorregulación, motivación, empatía y habilidades sociales. Para ambos instrumentos se utilizó la escala de Likert de 5 puntos, donde 1 significa "muy en desacuerdo"; 2 , "en desacuerdo"; 3 "indiferente"; 4 , "de acuerdo"; y 5, "muy de acuerdo". Los instrumentos empleados fueron aplicados de forma virtual a través de Google forms y para este fin se contó con la autorización de la Unidad de postgrado de la Facultad de Ciencias Administrativas de la Universidad Nacional Mayor de San Marcos.

La validación se llevó a cabo a través de juicio de expertos. Los resultados se sometieron a la prueba de KMO y Barlett cuyos indicadores fueron de 0.883 y 0.922 para las variables trabajo remoto y gestión de las emociones, respectivamente.

El Alfa de Cronbach fue utilizado en una prueba piloto aplicada a 63 estudiantes para determinar la confiabilidad de los instrumentos y se obtuvieron valores de 0.913 y 0.950 para trabajo remoto y gestión de las emociones, respectivamente. Estos resultados, demuestran que las herramientas aplicadas en el estudio tienen consistencia interna, por lo tanto, son confiables.

Los docentes investigadores y alumnos integrantes del equipo de investigación hicieron entrega del formulario de consentimiento informado a los encuestados. En algunos casos, dicho formulario fue proporcionado antes de completar el cuestionario.

\section{RESULTADOS}

Los resultados descriptivos se muestran a continuación (ver Figura 1):

Según la distribución etaria de la muestra, el 70\% de los encuestados se ubican en el segmento de edad comprendido entre 26 y 40 años, mientras que el $28 \%$ se ubica entre los 41 y 55 años.

En general, para la variable trabajo remoto, los resultados muestran que el $47 \%$ de los encuestados manifestó sentirse "indiferente" frente al trabajo remoto, el 33\% indicó estar "de acuerdo", mientras que el $18 \%$ se mostró "en desacuerdo", y solo el $2 \%$ y el $1 \%$ de encuestados, estuvo "muy de acuerdo" y "muy en desacuerdo", respectivamente.

En la Figura 1, se muestra la distribución de las dimensiones 1,2 y $3\left(D_{1}, D_{2}, D_{3}\right)$ de la variable trabajo remoto. Al respecto se observa que en relación a la dimensión jornada de trabajo $\left(D_{1}\right)$, el $41 \%$ de 


\section{Percepción de los encuestados sobre el trabajo}

\section{remoto}

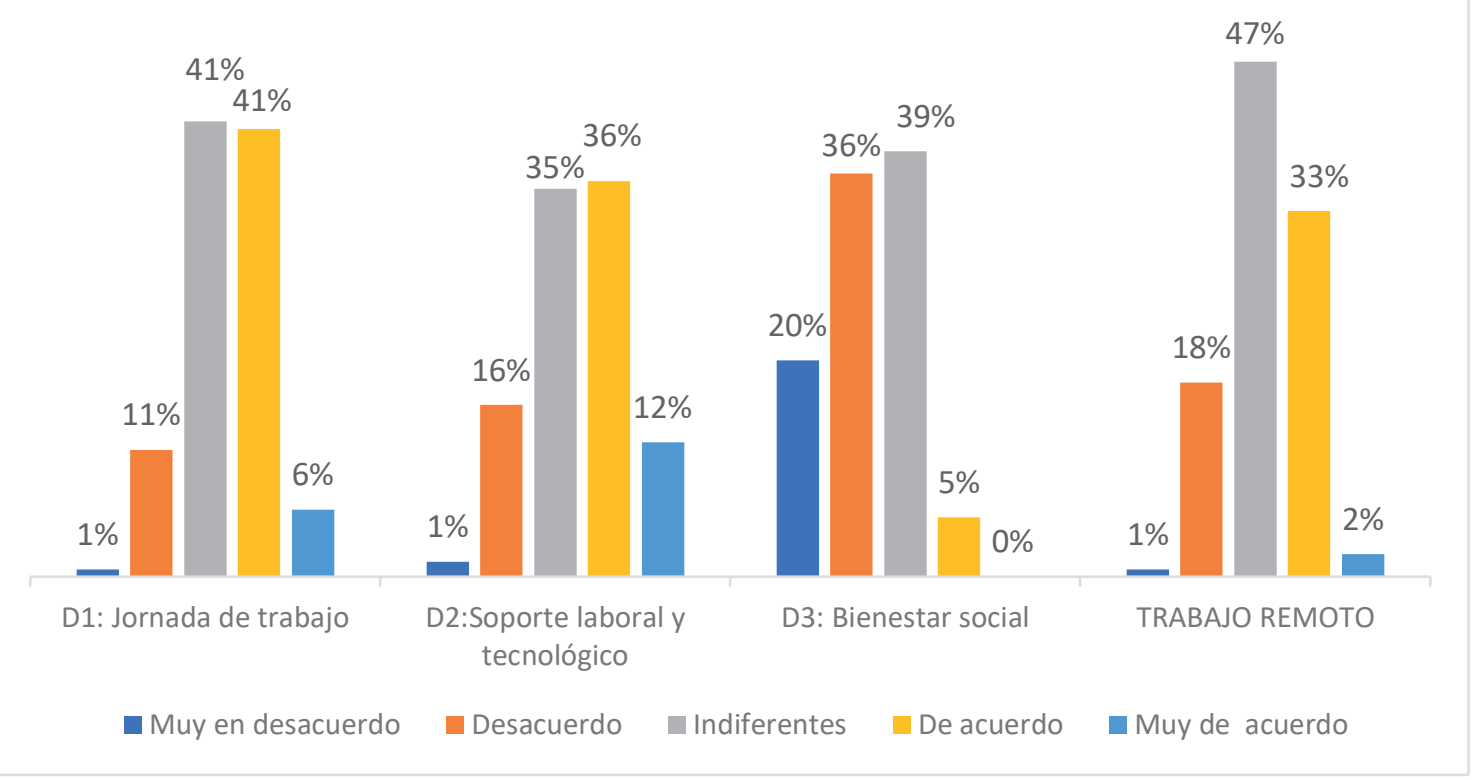

Figura 1. Gráfico de percepción de los trabajadores de dimensiones $D_{1}, D_{2}$ y $D_{3}$ de la variable Trabajo remoto.

Fuente: Elaboración propia obtenida de cuestionario aplicado a la muestra, año 2021.

encuestados señaló estar "de acuerdo" con esta, el mismo porcentaje se manifiesta "indiferente" respecto a esta variable, el $11 \%$ se mostró en "desacuerdo" y el $6 \%$ estuvo "muy de acuerdo" con la jornada de trabajo.

Respecto a la dimensión soporte laboral y tecnológico $\left(\mathrm{D}_{2}\right)$, el $36 \%$ de encuestados manifestó estar "de acuerdo" con el soporte laboral y tecnológico recibido, al $35 \%$ le resultó "indiferente", el 16\% indicó estar en "desacuerdo" y solamente el $12 \%$ consideró estar "muy de acuerdo" con el soporte laboral y tecnológico recibido de su organización.

Por otro lado, respecto a la tercera dimensión bienestar social $\left(D_{3}\right)$, el $39 \%$ de encuestados se mostró "indiferente", el $36 \%$ estuvo en "desacuerdo", el $20 \%$ "muy en desacuerdo" y el $5 \%$ señaló estar "de acuerdo". Estos resultados permiten afirmar que el $56 \%$ del total de encuestados manifestó estar entre "desacuerdo" y "muy en desacuerdo" con el servicio de bienestar social que le brindó su organización en el marco del trabajo remoto.

Finalmente, respecto a la variable gestión de las emociones, el $61 \%$ de encuestados se mostró "de acuerdo", el $22 \%$ indicó estar "muy de acuerdo", el
$14 \%$ es "indiferente", mientras que el $2 \%$ y el $1 \%$ manifestó estar en "desacuerdo" y "muy en desacuerdo" respectivamente (ver Figura 2).

\section{Prueba de hipótesis}

El trabajo remoto se relaciona con la gestión de las emociones en estudiantes de maestría de Administración con Mención en Dirección de Recursos Humanos, periodo 2020.

\section{Hipótesis}

La prueba de la hipótesis general de las variables trabajo remoto y la gestión de las emociones presenta el resultado siguiente:

$\mathrm{H}_{0}: \mathrm{r}=0$

No existe relación significativa entre el trabajo remoto y la gestión de las emociones.

$\mathrm{H}_{1}: \mathrm{r} \neq 0$

Existe relación significativa entre el trabajo remoto y la gestión de las emociones.

En la Tabla 1 se observa que existe una correlación positiva moderada de 0.465 con un nivel de signifi- 


\section{GESTIÓN DE LAS EMOCIONES}

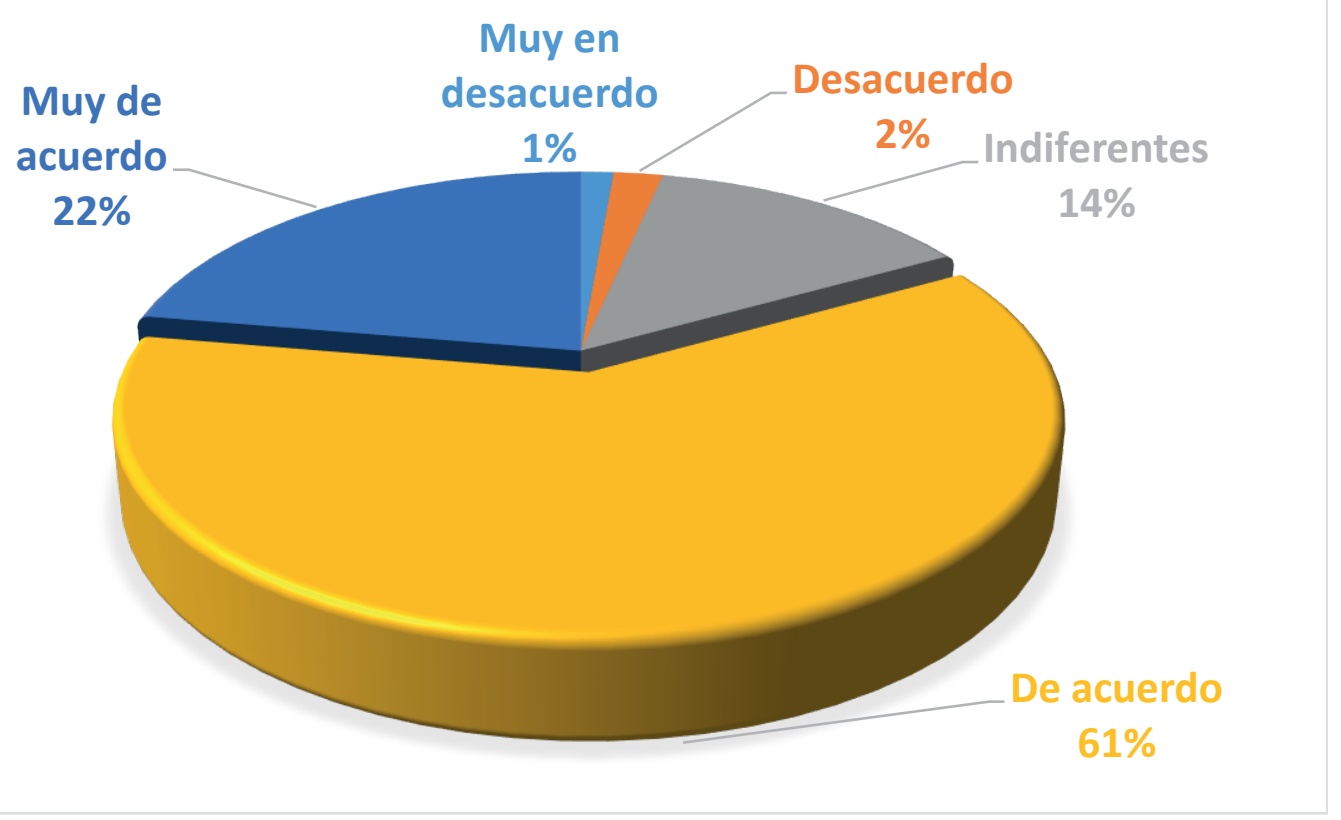

Figura 2. Gráfico de percepción de los trabajadores de la variable Gestión de las emociones.

Fuente: Elaboración propia a partir del cuestionario aplicado a la muestra, año 2021.

cancia de 0.001 entre el trabajo remoto y la gestión de las emociones en estudiantes de maestría de Administración con Mención en Dirección de Recursos Humanos.

Tabla 1. Valores Rho de Spearman para el trabajo remoto y la gestión de las emociones.

\begin{tabular}{|l|c|}
\hline & Valor \\
\hline Coeficiente de correlación & 0.465 \\
\hline $\mathrm{N}$ & 148 \\
\hline Sig. (bilateral) & 0.000 \\
\hline
\end{tabular}

Fuente: Elaboración propia a partir de resultados del software SPSS V25.

El resultado de las correlaciones de las dimensiones de la variable trabajo remoto $\left(D_{1}, D_{2}\right.$ y $\left.D_{3}\right)$, sometidas a la prueba de hipótesis específicas, con la variable gestión emocional, se muestra en la Figura 3. Se observa que existe una correlación positiva alta de 0.51 entre la dimensión jornada de trabajo $\left(D_{1}\right)$ y la variable gestión de las emociones en estudiantes de maestría en Administración con mención en Dirección de Recursos Humanos. Asimismo, existe una correlación positiva de 0.34 entre la dimensión soporte laboral y tecnológico
$\left(D_{2}\right)$ con la variable gestión de las emociones. Finalmente, existe una correlación positiva moderada de 0.37 entre la dimensión bienestar social $\left(D_{3}\right)$ y la variable gestión de las emociones. Las tres pruebas tienen un nivel de significación de 0.01 (ver Figura 3).

\section{DISCUSIÓN}

Los resultados de la investigación demuestran la existencia de una relación positiva entre el trabajo remoto y la gestión de las emociones en estudiantes de la maestría en Administración con mención en Dirección de Recursos Humanos, Lima 2020, la cual fue validada mediante la prueba de correlación Rho de Spearman.

En términos generales, las variables trabajo remoto y gestión de las emociones muestran resultados favorables. Si bien el $18 \%$ de la muestra está "en desacuerdo" con el trabajo remoto, esto se contrapone a un $33 \%$ que sí está "de acuerdo", sumado a un $47 \%$ que se mostró "indiferente". Es decir, los encuestados consideran que se han adaptado al trabajo remoto. Asimismo, el $86 \%$ se mostró entre "muy de acuerdo" y "de acuerdo" con el manejo de la gestión de sus emociones. Estos resultados se 


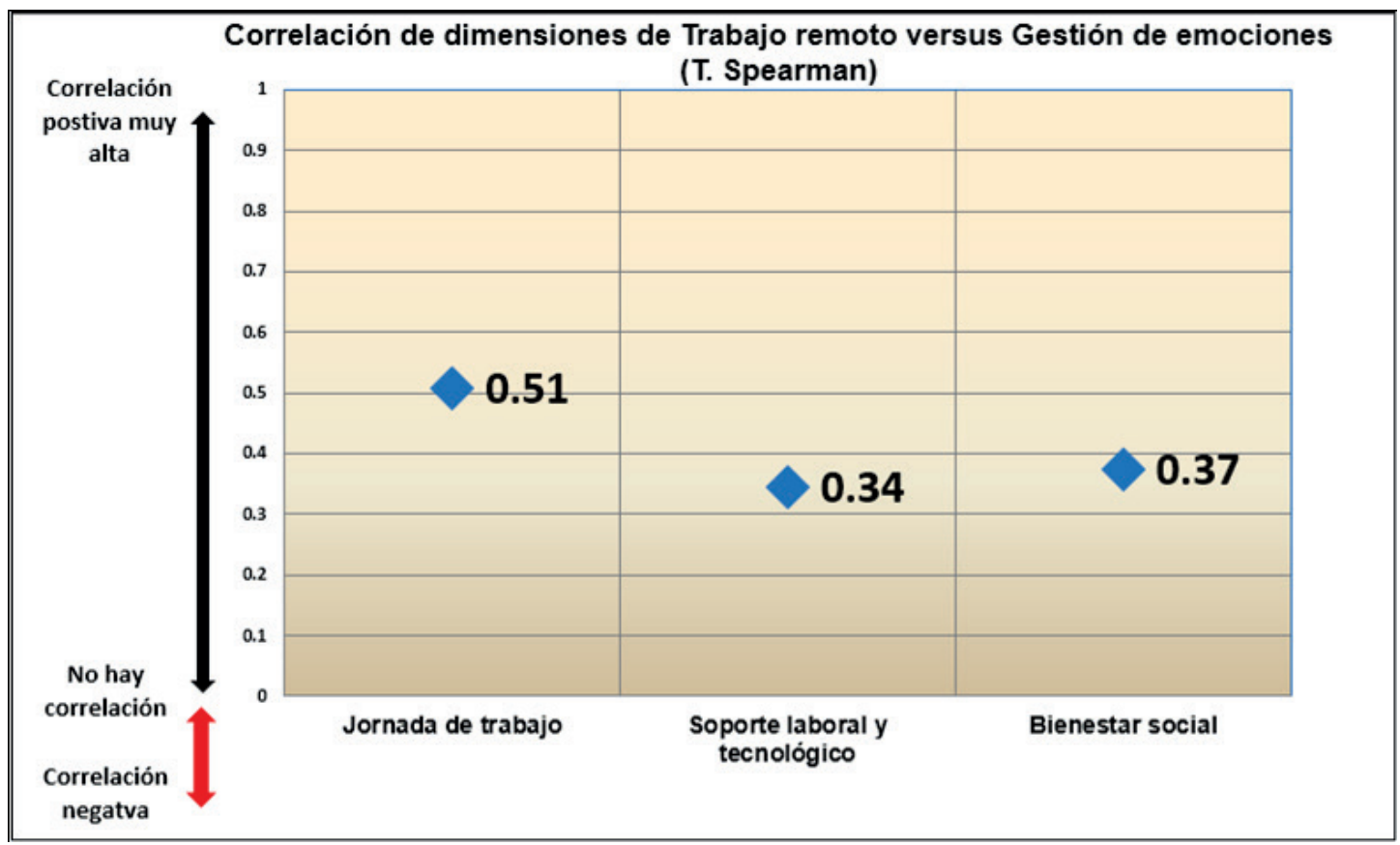

Figura 3. Gráfico de correlación de Spearman de las dimensiones de la variable trabajo remoto vs. gestión de las emociones.

Fuente: Elaboración propia a partir de resultados del software SPSS.

encuentran alineados con la investigación de Brinca y FutureLab citado por Chávez (2021), en el que se afirma que "el $49 \%$ señalan percibir emociones positivas, en tanto que el $24 \%$ afirman experiencias negativas y un $27 \%$ mantiene neutralidad, según el estudio, los resultados son bajos, ello debido a la carencia de políticas transparentes" [párr. 1].

Respecto a la primera dimensión de la variable trabajo remoto, la mayoría de los encuestados manifestaron sentirse "indiferentes" o "de acuerdo" con la jornada de trabajo establecida. El índice de correlación entre esta dimensión y la variable gestión de las emociones tiene un valor positivo y significativo de 0.51 . Esto supone que los encuestados aceptan la jornada de trabajo remoto y les resulta cómoda en términos generales. Estos resultados concuerdan con el estudio elaborado por Brinca y Futurelab citado por Crespo (2021), el cual señala que uno de sus hallazgos indica que el " $75 \%$ de los encuestados puede trabajar remotamente los cinco días de la semana, lo cual representa una buena señal de rápida adaptación" (párr. 10). Sin embargo, se considera que aún existen aspectos que no están siendo considerados por las organizaciones, tal como el reconocimiento de las horas extras o el exceso de trabajo. De acuerdo a los resultados de la presente investigación, el $71 \%$ de los encues- tados señaló no haber recibido pago adicional por las horas trabajadas fuera de la jornada de trabajo durante el tiempo que realizaron trabajo remoto.

En cuanto a la dimensión soporte laboral y tecnológico de la variable trabajo remoto, se observa que la mayoría de encuestados también señaló sentirse "indiferente" o "de acuerdo" respecto al apoyo que recibieron de parte de sus organizaciones; es decir, percibieron que sus organizaciones hicieron lo necesario para que las actividades laborales del trabajador no se vieran afectadas. Al respecto, se observa un nivel de correlación positiva y moderada de 0.34 con la variable gestión de las emociones. Evidentemente, el soporte laboral y tecnológico debe ir acompañado de reglas laborales claras para que tanto el trabajador como la organización se vean beneficiados tal como concluyen Peralta et al. (2020) al señalar que "considerando los elementos principales para la implantación de este sistema, como lo son las personas y la tecnología, aunado a la legislación, el Teletrabajo puede beneficiar tanto a las empresas como a sus trabajadores" (p. 334). En este sentido, el papel de las TIC juega un rol determinante en el trabajo remoto que debe ser visto como un instrumento que en realidad no desplaza al trabajador, sino más bien ayuda al logro de los objetivos (Buitrago, 2020). 
Finalmente, se observaron valores distintos en los resultados de la dimensión bienestar social de la variable trabajo remoto. La mayoría se mostró en "desacuerdo" o "muy en desacuerdo" con las políticas dirigidas a la atención del bienestar de los trabajadores y sus familias. Es decir, consideran que todos los aspectos vinculados a esta dimensión no han sido lo suficientemente atendidos por sus respectivas organizaciones; por tanto, los resultados arrojan una correlación positiva de 0.37 entre esta dimensión y la variable gestión de las emociones. En este sentido, la OIT (2020) en su "Guía práctica para el teletrabajo durante la pandemia de COVID-19 y después de ella" sugiere una serie de lineamientos para que el trabajador pueda realizar el trabajo remoto de tal forma que el impacto que supone mezclar los espacios laborales y familiares sea el menor posible.

\section{CONCLUSIONES}

La investigación demuestra que el trabajo remoto y la gestión de las emociones se relacionan de manera positiva. Se concluye que existe un nivel de correlación de 0.47 , lo que, junto con los datos descriptivos de ambas variables, sugiere que los encuestados, por un lado, se adaptaron rápidamente al trabajo remoto y, por otro, lograron un manejo efectivo de sus emociones.

La relación entre la dimensión jornada de trabajo y la variable gestión de las emociones es positiva y significativa con un valor de 0.51 . El análisis descriptivo indica que el $41 \%$ de los encuestados estuvo "de acuerdo" con la jornada del trabajo remoto, mientras que el mismo porcentaje se mostró "indiferente" respecto a esta; sin embargo, ambos grupos manifestaron que aún existen aspectos por mejorar en sus organizaciones.

El coeficiente de 0.34 obtenido señala que existe una correlación positiva entre el soporte laboral y tecnológico y la gestión de las emociones. Los resultados descriptivos muestran que el $36 \%$ de los encuestados estuvo de acuerdo y un $35 \%$ se mostró indiferente; solo el $17 \%$ señaló no estar conforme con el trabajo remoto. Se puede deducir que la mayoría de los encuestados tiene las herramientas necesarias para la gestión adecuada de su trabajo de manera remota.

El coeficiente de 0.37 obtenido señala que existe una correlación positiva entre la dimensión bienestar social y la gestión de las emociones. Sin embargo, los datos descriptivos indican que el 36\% señaló estar en desacuerdo con la manera cómo la organización maneja el bienestar social de los trabajadores durante el trabajo remoto. Resulta necesario que las organizaciones presten atención a esta dimensión, dado que además el bienestar social es un aspecto no visible en esta modalidad de trabajo.

De acuerdo a los resultados de esta investigación se observa que, a pesar de que no todos los aspectos del trabajo remoto se encuentran cubiertos, la mayoría de la población objeto de estudio integrada por estudiantes de la maestría de Recursos Humanos de la Universidad Nacional Mayor de San Marcos manifestó sentirse en una zona de confort respecto al trabajo remoto y gestionaron de manera eficiente sus emociones. Probablemente el hecho de que la mayoría de participantes del estudio pertenezca a la generación de los millennials, quienes se adaptan rápidamente a los cambios tecnológicos, personales y laborales, explica el bajo nivel de correlación entre ambas variables. Sin embargo, es necesario que las organizaciones públicas y privadas observen, rediseñen y comuniquen mejor aquellos elementos de la jornada de trabajo, del soporte laboral y tecnológico y del bienestar social que no se hayan considerado importantes. Es un hecho que la problemática será propia y distinta para cada organización.

Esta investigación deja abierta la posibilidad a nuevos estudios que incluyan otras variables como las brechas generacionales, diferencias de género, carga familiar, entre otros; no obstante, sirve de base para el estudio académico y sobre todo para que las organizaciones tomen mejores decisiones frente a esta nueva modalidad de empleo que probablemente se haya instaurado para quedarse por mucho tiempo.

\section{REFERENCIAS BIBLIOGRÁFICAS}

[1] Arbaiza, L. (2016). Cómo elaborar una tesis de grado. Lima, Perú: ESAN Ediciones

[2] Buitrago, D. (2020). Teletrabajo: una oportunidad en tiempos de crisis. CES Derecho, 11(1), 1-2. Recuperado de https://revistas.ces. edu.co/index.php/derecho/article/view/5620

[3] Cotrufo, T., y Ureña, J. (2018). El cerebro y las emociones: Sentir, pensar, decidir. Barcelona: Emse Edapp, S.L.

[4] Chávez, L. (12 de febrero de 2021). Trabajo remoto: ¿Por qué los trabajadores están insatisfechos y qué se puede cambiar para el 2021? El Comercio. Recuperado de https:// elcomercio.pe/economia/dia-1/trabajoremoto-por-que-los-trabajadores-no-estan- 
satisfechos-y-que-se-puede-cambiar-para-el2021-cuarentena-peru-noticia/

[5] Crespo, N. (19 de febrero de 2021): El 21\% de las personas trabajan remotamente de manera regular. FutureLab. Recuperado de https:// futurelab.pe/el-21-de-las-personas-trabajaremotamente-de-manera-regular/

[6] D. S. No. 017-2015-TR. Decreto Supremo que aprueba el Reglamento de la Ley $\mathrm{N}^{\circ}$ 30036, Ley que regula el teletrabajo. Diario Oficial El Peruano (2015). Recuperado de https://busquedas.elperuano.pe/download/url/ decreto-supremo-que-aprueba-el-reglamentode-la-ley-n-30036-decreto-supremo-n-0092015-tr-1307067-3

[7] D. S. No. 008-2020-SA (2020). Decreto Supremo que declara en Emergencia Sanitaria a nivel nacional por el plazo de noventa (90) días calendario y dicta medidas de prevención y control del COVID-19. Diario Oficial EI Peruano (2020). Recuperado de https://busquedas. elperuano.pe/download/url/decreto-supremoque-declara-en-emergencia-sanitaria-a-niveldecreto-supremo-n-008-2020-sa-1863981-2

[8] D. U. No. 026-2020. Decreto de Urgencia que establece diversas medidas excepcionales y temporales para prevenir la propagación del Coronavirus (COVID-19) en el territorio nacional. Diario Oficial El Peruano (2020). Recuperado de https://cdn.www.gob.pe/ uploads/document/file/566447/DU02620201864948-1.pdf

[9] D. U. N N 127-2020. Decreto de Urgencia que establece el otorgamiento de subsidios para la recuperación del empleo formal en el sector privado y establece otras disposiciones. Diario Oficial El Peruano (2020). Recuperado de https://cdn.www.gob.pe/uploads/document/ file/1473551/Decreto\%20de\%20Urgencia $\% 20$ N\%C2\%B0127-2020.pdf.pdf

[10] Ekman, P. (2013). El Rostro de las Emociones: Qué nos revelan las expresiones faciales. Barcelona: RBA Libros.

[11] Estrategias y Negocio (2021). El futuro del trabajo remoto en el Perú. Estrategias y Negocios. Recuperado de https://eyng.pe/ web/2021/02/11/el-futuro-del-trabajo-remotoen-el-peru/

[12] Eurofound, y Organización Internacional del Trabajo. (2019). Trabajar en cualquier momento y en cualquierlugar: consecuencias en elámbito laboral. Oficina de Publicaciones de la Unión
Europea, Luxemburgo y Oficina Internacional del Trabajo, Santiago. Recuperado de https://www.ilo.org/wcmsp5/groups/public/--americas/---ro-lima/---sro-santiago/documents/ publication/wcms_723962.pdf

[13] Gálvez, C. (2017). Inteligencia emocional. En F. Mureira (Ed.), ¿Qué es la inteligencia? (págs. 63-76). España: Bubok Publishing S.L.

[14] Gardner, H. (2014). Estructuras de la mente: La teoría de las inteligencias múltiples. Bogotá, Colombia: Fondo de Cultura Económica.

[15] Goleman, D. (1996). La inteligencia Emocional. Por qué es más importante que el cociente intelectual. Buenos Aires, Argentina.

[16] Johnson, M., Saletti-Cuesta, L., y Tumas, N. (2020). Emociones, preocupaciones y reflexiones frente a la pandemia del COVID-19 en Argentina. Ciência \& Saúde Coletiva, 25, 2447-2456. Recuperado de https://doi. org/10.1590/1413-81232020256.1.10472020

[17] Juárez, J. (2021). El futuro del Trabajo remoto en el Perú. Futurelab. Recuperado de https:// futurelab.pe/el-futuro-del-trabajo-remoto-en-elperu/

[18] Ley No. 30036 (15 de mayo de 2013). Ley que regula el Teletrabajo. Diario Oficial El Peruano (2013). Recuperado de https://busquedas. elperuano.pe/normaslegales/ley-que-regulael-teletrabajo-ley-n-30036-946195-3/

[19] López, J. E. (2020). Flexibilidad, protección del empleo y seguridad social durante la pandemia global del Covid-19. Documentos de Trabajo (IELAT, Instituto Universitario de Investigación en Estudios Latinoamericanos), 134, 1-74. Recuperado de https://dialnet.unirioja.es/ servlet/articulo?codigo $=7393704$

[20] Manucci, M. (2017). Competitividad emocional. Mercado. Recuperado de https://mercado.com. ar/management-marketing/competitividademocional/

[21] Ministerio de Justicia y Derechos Humanos y SERVIR (2016). Guía sobre el sistema administrativo de Gestión de Recursos Humanos en el Sector Público. Guía para asesores jurídicos del Estado. Recuperado de https://www.minjus.gob. pe/wp-content/uploads/2016/07/MINJUSDGDOJ-Gu\%C3\%ADa-sobre-el-SistemaAdministrativo-Servir.pdf

[22] Ministerio de Trabajo y Promoción del Empleo (2019) Manual de preguntas frecuentes 
laborales. Recuperado de https://cdn.www.gob. pe/uploads/document/file/307150/MANUAL_ DE_PREGUNTAS_FRECUENTES.pdf

[23] Organización Internacional del trabajo OIT (1966). C177 - Convenio sobre el trabajo a domicilio, 1996 (núm. 177). Recuperado de https://www.ilo.org/dyn/normlex/es/f?p=NO RMLEXPUB:12100:0::NO::P12100_ILO_ CODE:C177

[24] Organización Internacional del Trabajo OIT (2020). El teletrabajo durante la pandemia de COVID-19 y después de ella. Guía práctica. Recuperado de https://www.ilo.org/wcmsp5/ groups/public/---ed_protect/---protrav/---travail/ documents/publication/wcms_758007.pdf

[25] Organización Mundial de la Salud (2020). COVID-19: Cronología de la actuación de la OMS. Recuperado de https://www.who.int/es/ news/item/27-04-2020-who-timeline---covid-19
[26] Peralta, A., Bilous, A., Flores, C., y Bombón, C. (2020). El impacto del teletrabajo y la administración de empresas. Recimundo, 4(1), 326-335. Recuperado de https:// doi.org/10.26820/recimundo/4.(1). enero.2020.326-335

[27] Rivas, L. A. (2017). Elaboración de Tesis. Estructura y Metodología. Ciudad de México, México: Editorial Trillas.

[28] Salovey, P, y Mayer, J. D. (1990). Emotional Intelligence. Imagination, Cognition and Personality, 9(3), 185-211. Recuperado de https://doi.org/10.2190/DUGG-P24E-52WK6CDG

[29] Weller, J. (2020). La pandemia del COVID-19 y su efecto en las tendencias de los mercados laborales. Santiago, Chile: CEPAL. Recuperado de https://repositorio.cepal.org/bitstream/ handle/11362/45759/1/S2000387_es.pdf 


\title{
Remote Work and Emotion Management in Times of COVID-19: A Perspective of Master's Degree Students as Workers, Lima-Peru (2020)
}

\author{
Justina Uribe KaJat ${ }^{1}$ \\ Kelly Myriam Jiménez de Aliaga ${ }^{2}$ \\ José Gerardo VARGas DÁVILA ${ }^{3}$ \\ Daniel Enrique Rey de Castro Hidalgo ${ }^{4}$ \\ Miguel Ángel Bashualdo Pérez ${ }^{5}$ \\ Luis Alberto Geraldo Campos ${ }^{6}$
}

Received: 15/03/2021 Accepted: 24/04/2021 Published: 26/07/2021

\begin{abstract}
The main objective of this study is to determine the relationship between remote work and emotion management. It also aims to determine the relationship between the dimensions "working hours", "work and technological support" and "social wellness", and the emotion management variable. A methodology based on a quantitative approach with a descriptivecorrelational and cross-sectional scope was used on a sample of 148 master's degree students of the School of Administration of Universidad Nacional Mayor de San Marcos. Likert-type scale surveys were used to gather data. The methods of analysis used were descriptive and correlational. The results indicate that the variables remote work and emotion management are positively and moderately correlated (0.465); therefore, it is concluded that remote work and all its dimensions are related to emotion management.
\end{abstract}

Keywords: remote work; emotion management; master's degree students; workers; COVID-19.

\section{INTRODUCTION}

Humanity is currently experiencing a crisis as a result of the COVID-19 health emergency, which is defined by the World Health Organization as a pandemic (2020) that has changed the routine and daily life of people. The world of work as we conceive it has changed; in Europe, according to the International Labor Organization (ILO), "The most significant increase in teleworking took place in countries that were most affected by the virus, and where teleworking was well developed before the pandemic" (2020, p. 3); in Peru, legislation, albeit incipient, on teleworking already existed.

As of the first week of March 2020, data on the behavior of COVID-19 were available in Europe, mainly in Italy, France and Spain, where the pandemic was already leaving deadly consequences. In its chronology of COVID-19 performance on March 11, 2020, WHO reported that it was "Deeply concerned by the alarming levels of spread and severity, and by the alarming levels of inaction" and determined in its assessment that "COVID-19 can be char-

$1 \mathrm{PhD}$ in Administration and in Education from Universidad Nacional Federico Villarreal (Lima, Peru). Currently working as professor at the same university. (Lima, Peru).

ORCID: https://orcid.org/0000-0002-2691-3073

E-mail: juribe@unmsm.edu.pe

$2 \mathrm{PhD}$ in Nursing from Universidade Federal do Río de Janeiro (Rio de Janeiro, Brasil). Research professor at Universidad Cesar Vallejo. (Lima, Peru). ORCID: https://orcid.org/0000-0002-8959-265X E-mail: kmjimenez@ucv.edu.pe

3 Master in Public Management. Currently working as professor at Universidad Nacional Mayor de San Marcos. (Lima, Peru).

ORCID: https://orcid.org/0000-0002-4914-8108

E-mail: jvargasda@unmsm.edu.pe

4 Master in Business Management. Currently working as an independent consultant. (Lima, Peru)

ORCID: https://orcid.org/0000-0002-0610-2150

Corresponding author: danielreydecastro@gmail.com

5 Degree in Administration from Universidad Nacional Federico Villareal. Specialist in Human Capital Management. Currently working as consultant at the Ministerio Público del Perú. (Lima, Peru).

ORCID: https://orcid.org/0000-0001-6456-8592

E-mail: miguelj1528@gmail.com

6 Master in Business Management and Administration. Currently working as professor at Universidad Peruana Unión. (Lima, Peru).

ORCID: https://orcid.org/0000-0002-8366-689X

E-mail: luis.geraldo@upeu.edu.pe 
acterized as a pandemic" (para. 20). From this date onwards, most of the world's governments adopted measures to prevent the spread of the virus in their countries.

In Peru, in response to the COVID-19 pandemic, a Health Emergency was declared on March 11, 2020 throughout the national territory for a period of 90 calendar days through Supreme Decree No. 008-2020-SA. Likewise, under this legal norm, prevention and control measures were adopted for all sectors of public and private activity.

Therefore, the Peruvian Government, through Emergency Decree No. 026-2020, among other measures, established remote work in order to safeguard the safety and health of workers; consequently, a new way of working was established, different from what we were used to. This alarming situation, as of that official moment in the country, caused uncertainty in companies and forced them to rethink their processes in the midst of social isolation. In this regard, Juárez (2021) stated that COVID-19 "tomó a las organizaciones por sorpresa, en pleno proceso de implementación de su transformación digital, mientras que otras aún no tenían al trabajo remoto como una opción [took organizations by surprise, in the middle of their digital transformation, while others still had not yet considered remote work as an option]" (para. 2). This scenario ultimately became an irrefutable reality.

The ILO Convention 177 (1966) on home work is a precedent of telework and remote work, which established that home work refers to work performed at home or in any other place other than the one offered by the employer; in this context, workers receive remuneration, provide a service or produce a product according to the requirements of the employer, who is a legal person. This supranational standard urges States to protect all workers, i.e., to preserve all labor rights. It should be noted that the use of information and communication technologies is not mentioned in any of the provisions of the aforementioned agreement. To date, this agreement has only been ratified by ten countries, among which Peru is not included.

In this context, Eurofound and ILO (2019) in their report "Working anytime, anywhere: The effects on the world of work" present the results of a research carried out in 15 countries observed ${ }^{7}$; referring to

7 Countries observed are: Argentina, Brazil, India, Japan, the United States, Belgium, Finland, France, Germany, Hungary, Italy, Netherlands, Spain, Sweden and Great Britain
telework/ICT-mobile work (T/ICTM) ${ }^{8}$, they point out that ICT have revolutionized work and people's lives and have enabled real-time connection with families, as well as with immediate bosses and co-workers. They also consider that the negative aspect of this new way of working is "the encroachment of paid work into the spaces and times normally reserved for personal life" (p. 8); however, they also observe that technological advances are favorable for employees because they promote "spatial and temporal flexibility, in order to help them to balance work demands with their family and other personal responsibilities" (p.62). Similarly, the results of the T/ICTM are positive for the employers, as they allowed them to achieve better performance and optimize productivity.

Information and Communication Technologies (ICTs) have transformed business activity as well as people's lives. In developed countries, a significant progress had already been made regarding teleworking and other remote modalities; however, in countries such as Peru, where ICTs were still in their early stages of development, work systems had to be implemented according to their own reality. Under these circumstances, the Peruvian government opted to establish remote work.

At present, the terms telework and remote work are used interchangeably as if they had the same concept and application; nevertheless, they are different. Under Peruvian law, telework is regulated by Law No. 30036, D. S. No. 017-2015-TR, which essentially establishes that the acceptance of the worker is required for its implementation, work is subordinated and does not require the worker's physical attendance. This regulation applies to the public and private sectors as it is a modality in which the labor relationship is maintained and carried out using ICT in its different formats, and the employer exercises labor control and supervision through it. Subordination is further evidenced by the provision of IT tools and other methods to assist in the performance of the function.

Conversely, remote work is regulated by Emergency Decree No. 026-2020 and its implementation is not freely chosen by the worker. The pandemic forced its implementation in order to prevent the spread of the disease among the population; for this reason, remote work is performed at the worker's home or at the place of home isolation known by the employer. Accordingly, measures were

8 In the study, T/ICTM is defined the use of ICTs, including computers, mobiles, laptops, to provide services as employees outside the premises of the employer's company. 
adopted to guide employers to prevent affecting, among other aspects, the employment relationship and remuneration, and also to keep them informed on the health and safety measures to be followed. Guidelines on information security and confidentiality, compliance with health and safety measures in the place of isolation, and availability during the working hours were also issued for workers. As in teleworking, ICTs or other means are used for the fulfillment of functions insofar as permitted by their nature.

Remote work is thus a brand-new work system that emerged as a consequence of the COVID-19 pandemic and, to date, the effects or relationship between remote work and the management of workers' emotions are unknown, even though there are some studies and research related to teleworking and its effect on emotions.

Weller (2020), referring to telecommuting and teleworking in the context of the pandemic, observes that digitalization has assisted the labor scenario and has contributed to achieve "conciliación entre el trabajo y la vida familiar, la descongestión del tráfico urbano [work-life balance, and to reduce urban traffic congestion]" (p. 12); he further states that the increase of jobs supported by digitized platforms is expected at a global level. Weller (2020) and López (2020) agree that telecommuting in times of COVID-19 helps to prevent the spread of the pandemic in the workplace and, correspondingly, at home.

It is believed that as long as the pandemic continues, and probably after it, face-to-face work will be necessary in specific cases such as production centers, the agricultural industry, mines, among other economic activities; however, remote work is a modality that has not been discarded as a permanent option over time.

Peralta et al. (2020) analyze the impact of teleworking on companies as a result, primarily, of the advance of ICT. According to them, it has had an impact on the labor area and has forced companies to adopt new ways of working such as teleworking. They state that teleworking "rompe los esquemas tradicionales y genera en el teletrabajador mayor confianza en la organización y sentido de pertenencia, lo que persigue y consecuentemente espera una mayor productividad y eficiencia en los procesos administrativos [challenges traditional schemes and generates in the teleworker greater confidence in the organization and a sense of belonging, which is expected to increase productivity and efficiency in administrative processes]" (p. 9), something that can be achieved because this modality is part of labor flexibility.

A series of reflections on emotions in the context of the COVID-19 pandemic are provided by Johnson et al. (2020), who conclude that there is "la necesidad de diseñar estrategias para disminuir la incertidumbre con el objetivo de mejorar la salud de la población, considerando las desigualdades sociales y de género existentes [a need to design strategies to reduce uncertainty in order to improve the health of the population, taking into account existing social and gender inequalities]" (p. 2455). These reflections should be applied to all workers, regardless of the activity they carry out.

There is concern about the COVID-19 pandemic not only among governments, but also among international organizations such as WHO and ILO. Each has issued standards for governments to adopt according to their circumstances. Vulnerability is not the same in all countries, and the measures taken are based on scientific and technological advances.

In the publication of the supplement Día1 of the Peruvian newspaper El Comercio on remote work, Chávez (2021) reports positive results obtained by FutureLab on the management of emotions during this health emergency period. An important aspect addressed was the need for companies to ensure the safeguarding of the health of their employees' families. This stance is also in line with the conclusions of an article recently published by Estrategias y Negocios (2021).

This research addresses two variables: remote work and emotion management. Remote work was approached considering the worker's right to digital disconnection and to respect working hours (D. U. No. 127-2020); it was also analyzed based on three dimensions: working hours, work and technological support, and social wellness.

Working hours are understood as "el tiempo que cada trabajador dedica a la ejecución del trabajo para el que ha sido contratado. Esta jornada tiene un límite establecido por la Ley que es de 8 horas diarias o 48 horas semanales, como máximo [the time each worker devotes to the execution of the work for which he/she has been hired. By law, the limit of this working hours is established to be 8 hours a day or 48 hours a week, at most]" (Ministerio de Trabajo y Promoción del Empleo, 2019, p. 10); technological work support should be understood as support activities for workers managed by entities in times of pandemic and evidenced in guidelines for the optimal development of their functions, 
the transfer of information, technological programs, equipment and other tools that enable remote work. Finally, social wellness comprises activities aimed at creating an adequate work environment and a better quality of life for employees (MINJUS-SERVIR, 2016).

Analysis of emotional intelligence urges researchers to evoke the contributions of Gardner (2014), who considers that intelligences are intellectual capacities or domains that use specific skills for specific purposes, which gives rise to the theory of multiple intelligences. Based on this theory, and in particular on the types of intrapersonal and interpersonal intelligence, Salovey and Mayer (1990) first proposed the concept of emotional intelligence with emphasis on the cognitive processes of emotional evaluation and expression, as well as emotional regulation.

Regarding emotions in a critical pandemic situation, Ekman (2013) states that they are of valuable significance for life, because they allow us to feel pleasure and externalize joy, as well as to experience other types of feelings such as anger, hatred or jealousy; all of them, generated by external stimuli. As Cotrufo and Ureña (2018) pointed out, emotions have an extremely important function in changing contexts of crisis and uncertainty similar to those we are living in these times, and that is adaptation, as Charles Darwin stated in 1872 in a publication he titled The Expression of Emotions in Man and Animals.

Under these circumstances, it is important to focus on the impact of the pandemic on emotions, as pointed out by Johnson et al. (2020) when they state that it is essential to concentrate on mental health "no solo para mejorar la salud sino también para evitar otros problemas sociales, tales como la estigmatización de personas, la falta de adherencia a medidas de prevención, y el duelo frente a la pérdida de seres queridos [not only to improve health but also to avoid other social problems, such as personal stigmatization, lack of adherence to prevention measures, and coping with grief at the loss of loved ones]" (p. 2448). They agree with Manucci (2017) that the development of people's emotional capacity cannot be postponed, considering that people who perform remote work are no strangers to the emotional impact of the pandemic.

To understand emotional management, we must start by knowing what emotions are. Goleman (1996) stated that "emotions are, in essence, impulses to act, the instant plans for handling life that evolution has endowed instilled in us [...] suggesting that in every emotion there is an implicit tendency to act" (p.24), and that emotion "is any feeling, thought, psychological or biological state unique to it and a part of personal tendency to act accordingly" (p.331). He further noted that there are some primary emotions: anger, sadness, fear, pleasure, love, surprise, disgust, and shame. From neuroscience, Cotrufo and Ureña (2018) consider that emotions "son un conjunto de cambios que se dan a nivel fisiológicos, cognitivos, subjetivos y motores [are a set of changes that occur at physiological, cognitive, subjective and motor levels]" (p.18) and arise from a conscious or unconscious perception based on the circumstances and the concrete reality (stimuli) in which a person interacts.

According to Goleman (1996), self-awareness is the ability to recognize personal emotions and identify a feeling as it occurs; self-regulation consists of the ability of people to adequately channel their feelings, based on the awareness of their own being; and motivation is related to the ability to adequately structure emotions to better focus and prioritize objectives. In turn, empathy refers to the ability to recognize and understand others' emotions, which, in a way, allows people to adapt to crisis contexts such as the one caused by the coronavirus pandemic. Finally, social skills are related to the ability to properly manage interactions with others. Consequently, as noted in Gálvez (2017), emotional management is the ability of people to regulate emotions and adapt to changes that may occur in their environment of interaction.

This research therefore seeks to answer the following question: what is the relationship between remote work and emotion management? Its general objective is to determine the relationship between remote work and the management of emotions of the students of the Master in Administration with Mention in Human Resources Management, as workers in the public or private sector. It also aimed to determine the relationship between the dimensions of working hours, technological work support and social wellness, and the variable of emotion management.

The pandemic situation the world is going through has undoubtedly led to new forms of coexistence commonly known as the "new normal", where physical distance is one of the fundamental rules. Most governments were forced to introduce remote work, a form of telecommuting imposed and implemented without a legal framework or sufficiently solid technological support in many countries, among them Peru. Thus, considering human capital as the most important intangible element in organizations, it is important to study the impact of remote work on 
workers on the basis of the fundamental dimensions for good work performance. This research explores a new and under-studied topic as a result of the rapid and surprising spread of the COVID-19 pandemic and its consequences on the planet. Remote work is very recent in Peru and, as such, it raises new challenges for public and private organizations and entities regarding the implementation of effective management strategies that guarantee, on the one hand, the productivity and optimal performance of workers, and on the other hand, their wellness and emotional health. This research also contributes to knowledge, mainly on the perception and behavior of workers when confronted with critical and unexpected situations that require the introduction of new work modalities. Finally, this research may serve as a basis for future academic studies.

\section{MATERIALS AND METHODS}

This study follows a descriptive approach for, as Rivas (2017) points out, it "describe al sujeto de investigación, sin hacer juicios de valor sobre él pero analizando las descripciones y buscando asociaciones entre ellas [describes the research subject, without making value judgments about it while analyzing the descriptions and looking for associations between them]" (p.128). It is correlational because, according to the same author, "buscan medir el grado en que están asociadas dos o más variables [they seek to measure the degree to which two or more variables are related]" (p.132). It is cross-sectional because it focuses on obtaining data on a certain event at a certain time (Arbaiza, 2016).

The sample consisted of 148 participants of the Master's in Administration with mention in Human Resources Management, academic period 2020, of the Postgraduate Unit of the School of Administrative Sciences of the UNMSM, Lima, Peru.

Two instruments were applied to the respondents. The first corresponded to remote work variable and included 26 items distributed in three dimensions: working hours, work and technological support, and social wellness; the second was designed to measure emotion management variable and included 20 items distributed in five dimensions: self-awareness, self-regulation, motivation, empathy and social skills. A 5-point Likert scale was used for both instruments, where 1 means "strongly disagree"; 2 , "disagree"; 3, "indifferent"; 4, "agree"; and 5, "strongly agree". Instruments were applied via Google forms, with the authorization of the Postgraduate Unit of the School of Administrative Sciences of the UNMSM.
Expert judgment was used for data validation. Results were subjected to the KMO and Barlett's tests yielding 0.883 for remote work and 0.922 for emotion management.

Cronbach's alpha was used in a pilot test applied to 63 students to determine the reliability of the instruments and obtained values of 0.913 for remote work and 0.950 for emotion management. Results show that the tools applied in the study have internal consistency and are, therefore, reliable.

Research professors and student members of the research team handed out the informed consent form to the respondents. In some cases, this form was provided before completing the questionnaire.

\section{RESULTS}

Descriptive results are shown below (see Figure 1):

Based on the age distribution of the sample, $70 \%$ of the respondents ranged between ages $26-40$, while $28 \%$ ranged between ages 41-55.

For the remote work variable, $47 \%$ of respondents were "indifferent" towards remote work, 33\% "agreed" with remote work, 18\% "disagreed" with remote work, and only $2 \%$ and $1 \%$ of respondents "strongly agreed" and "strongly disagreed" with it, respectively.

The distribution of dimensions $D_{1}, D_{2}$, and $D_{3}$ of the remote work variable is shown in Figure 1. It is observed that $41 \%$ of respondents "agreed" with working hours dimension $\left(D_{1}\right)$, the same percentage was "indifferent" to it, 11\% "disagreed" and 6\% "strongly agreed" with working hours dimension.

As for work and technological support dimension $\left(D_{2}\right), 36 \%$ of respondents declared that they "agreed" with the work and technological support received, $35 \%$ were "indifferent", $16 \%$ indicated that they "disagreed" with it and only $12 \%$ considered that they "strongly agreed" with the work and technological support received from their organization.

Regarding the social wellness dimension (D3), $39 \%$ of respondents were "indifferent" towards it, $36 \%$ "disagreed", 20\% "strongly disagreed" and 5\% "agreed" with it. These results show that $56 \%$ of all respondents stated either "disagree" or "strongly disagree" with the social wellness support provided by their organization in the context of remote work.

Lastly, for the variable emotion management, $61 \%$ of respondents "agreed" with emotion management, $22 \%$ "strongly agreed" with it, $14 \%$ were "indifferent" 


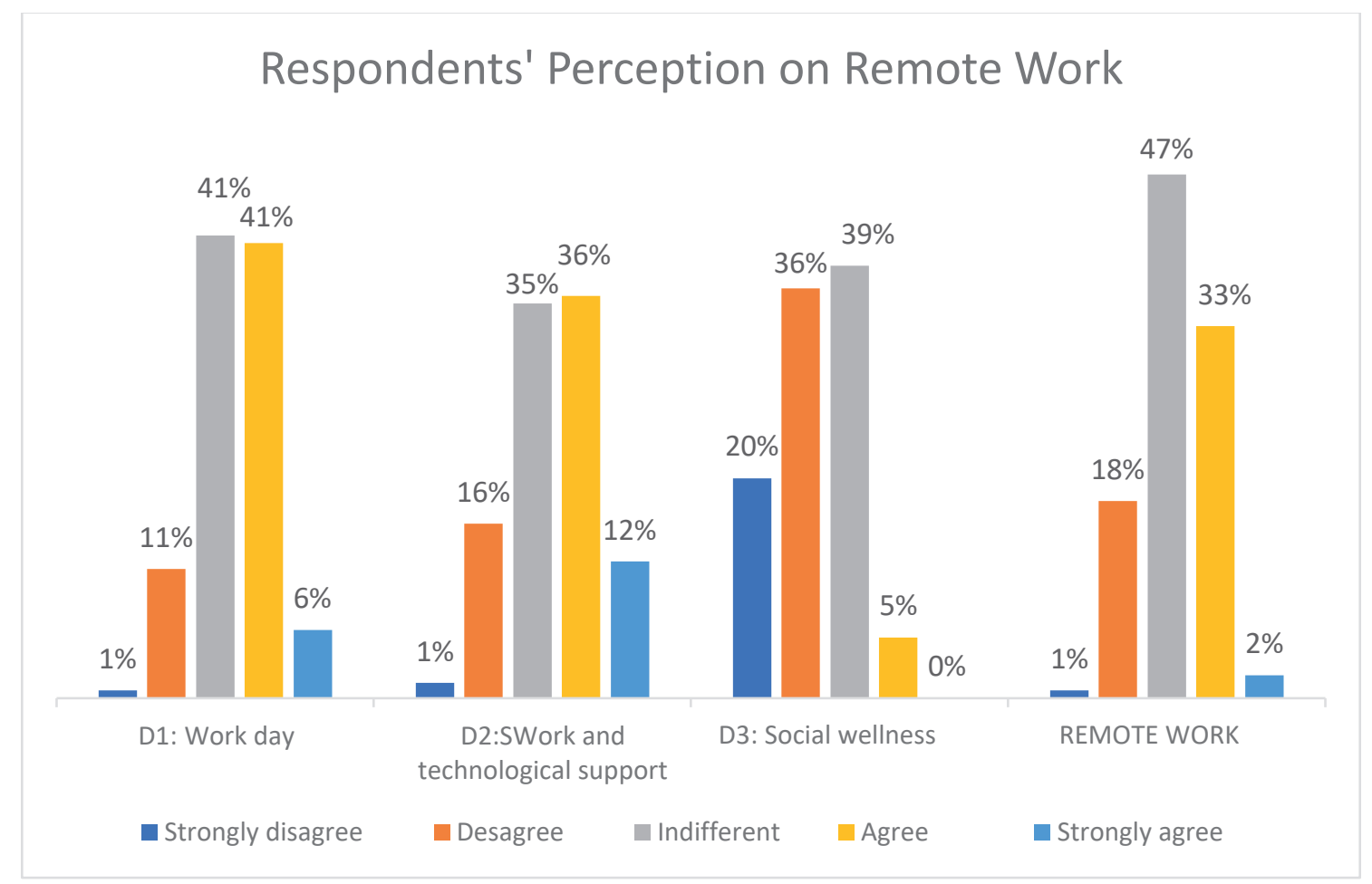

Figure 1. Chart showing workers' perception on variable remote work dimensions $D 1, D 2$ and D3.

Source: Prepared by the authors based on the questionnaire applied to the sample, 2021.

towards it, while $2 \%$ and $1 \%$ stated they "disagreed" and "strongly disagreed" with it, respectively (see Figure 2).

\section{Hypothesis Testing}

A relationship exists between remote work and emotion management of students of Master's in Administration with Mention in Human Resources Management, academic period 2020.

\section{Hypothesis}

General hypothesis testing for the variables remote work and emotion management yielded the following result:

$\mathrm{H}_{0}: \mathrm{r}=0$

There is no significant relationship between remote work and emotion management.

$\mathrm{H}_{1}: r \neq 0$

There is a significant relationship between remote work and emotion management.

Table 1 shows that there is a moderate positive correlation of 0.465 with a significance vel of 0.001 between remote work and emotional management in students of Master's in Administration with Mention in Human Resources Management.

Table 1. Spearman's Rho Values for Remote Work and Emotion Management.

\begin{tabular}{|l|c|}
\hline & Value \\
\hline Correlation Coefficient & 0.465 \\
\hline $\mathrm{N}$ & 148 \\
\hline Sig. (2-tailed) & 0.000 \\
\hline
\end{tabular}

Source: Prepared by the authors based on results from SPSS V25 software.

Figure 3 shows the results of the correlations of the dimensions of remote work variable $\left(D_{1}, D_{2}\right.$ and $\left.D_{3}\right)$, subjected to the specific hypothesis testing, with emotional management variable. A high positive correlation of 0.51 is observed between working hours dimension $\left(D_{1}\right)$ and emotion management variable in students of Master's in Administration with Mention in Human Resources Management. In addition, there is a positive correlation of 0.34 between work and technological support dimension $\left(D_{2}\right)$ and emotion management variable. Finally, there is a moderate positive correlation of 0.37 between social wellness dimension $\left(D_{3}\right)$ and emotion 


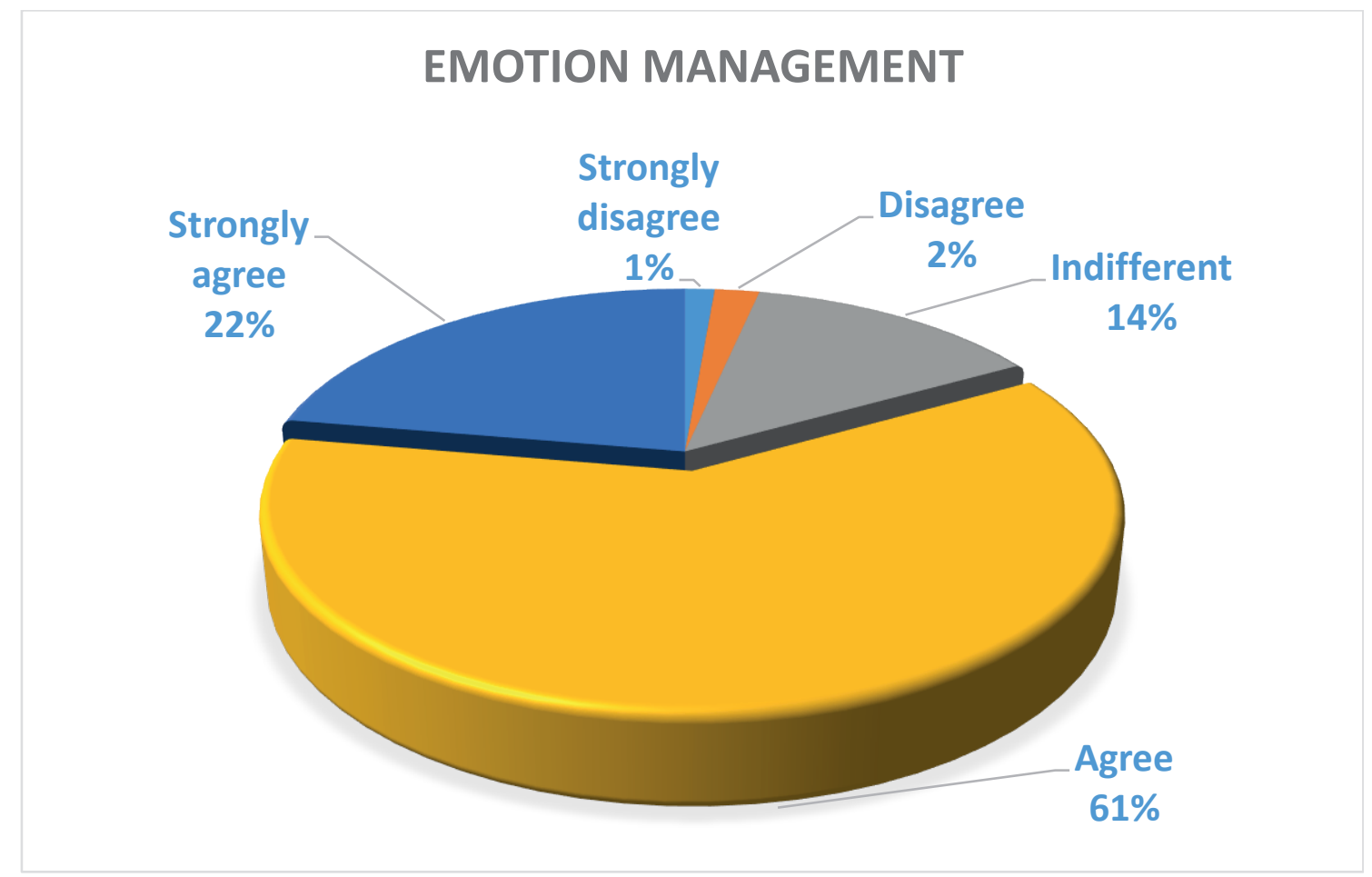

Figure 2. Chart showing workers' perception on variable emotion management.

Source: Prepared by the authors based on the questionnaire applied to the sample, 2021.

management variable. All three tests have a significance level of 0.01 (see Figure 3).

\section{DISCUSSION}

Research results demonstrate the existence of a positive relationship between remote work and emotion management in students of Master's in Administration with Mention in Human Resources Management, Lima 2020, which was validated using Spearman's Rho correlation test.

On the whole, the variables remote work and emotion management show favorable results. Although $18 \%$ of the sample "disagreed" with remote work, there were $33 \%$ who "agreed" and $47 \%$ who were "indifferent". In other words, respondents believe that they have adapted to remote work. Likewise, $86 \%$ of respondents said they "strongly agree" or "agree" with the way they manage their emotions. These results coincide with the research by Brinca and FutureLab cited by Chávez (2021), which states that "el $49 \%$ señalan percibir emociones positivas, en tanto que el $24 \%$ afirman experiencias negativas y un $27 \%$ mantiene neutralidad, según el estudio, los resultados son bajos, ello debido a la carencia de políticas transparentes [49\% of respondents experienced positive emotions, $24 \%$ reported negative experiences and $27 \%$ maintained neutrality, according to the study, results are low due to the lack of transparent policies]" [para. 1].

Regarding the first dimension of the remote work variable, most respondents reported being "indifferent" or "in agreement" with the established working hours. There is a positive and significant correlation coefficient of 0.51 between this dimension and emotion management variable. This implies that respondents accept the remote working hours and find it comfortable in general terms. These results agree with the study by Brinca and Futurelab cited by Crespo (2021), which points out that " $75 \%$ de los encuestados puede trabajar remotamente los cinco días de la semana, lo cual representa una buena señal de rápida adaptación [75\% of respondents can work remotely five days a week, a good sign of rapid adaptation]" (para. 10). However, there are still aspects that organizations do not consider, such as the recognition of overtime or overwork. The results of our research show that $71 \%$ of respondents reported not having received additional payment for overtime while working remotely. 


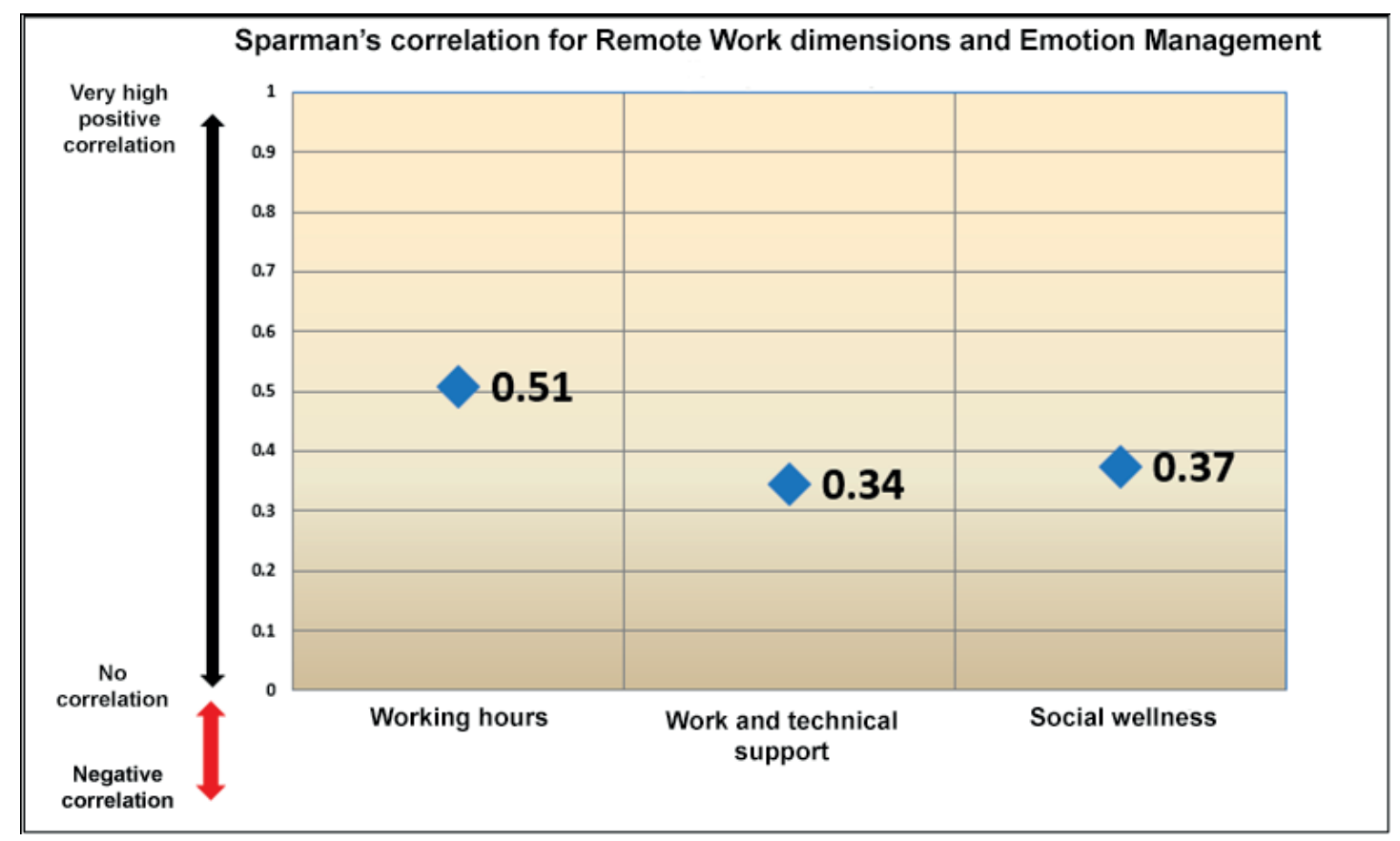

Figure 3. Spearman correlation chart showing the dimensions of remote work vs emotion management.

Source: Prepared by the authors based on results from SPSS V25 software.

Regarding labor and technological support dimension of the remote work variable, most respondents also reported being "indifferent" or "in agreement" with respect to the support they received from their organizations; that is, they perceived that their organizations did what was necessary so that the worker's work activities would not be affected. A moderate positive correlation coefficient of 0.34 was observed between this dimension and emotion management variable. Clear labor rules must accompany work and technological support so that both the worker and the organization benefit from it, as concluded by Peralta et al. (2020) by stating that "considerando los elementos principales para la implantación de este sistema, como lo son las personas y la tecnología, aunado a la legislación, el Teletrabajo puede beneficiar tanto a las empresas como a sus trabajadores [considering the key elements for the implementation of this system, such as people and technology, together with the legislation, teleworking can benefit both companies and their workers]" (p. 334). In this sense, ITCs play a decisive role in remote work, and should be seen as an instrument that in fact does not replace the worker, but rather assists in the achievement of objectives (Buitrago, 2020).

Finally, different values were observed for social wellness dimension of the remote work variable.
Most respondents "disagreed" or "strongly disagreed" with the policies aimed at the well-being of workers and their families. That is, they consider not enough attention was paid to all aspects related to this dimension by their respective organizations; therefore, the results show a positive correlation of 0.37 between this dimension and emotion management variable. In this regard, the ILO (2020) in Teleworking during the COVID-19 pandemic and beyond, A Practical Guide suggests a series of guidelines designed to enable the worker to perform remote work while minimizing the negative impact of mixing work and family spaces.

\section{CONCLUSIONS}

The research shows that remote work and emotion management are positively related. A correlation level of 0.47 is concluded, which, together with the descriptive data of both variables, suggests that respondents, on the one hand, quickly adapted to remote work and, on the other hand, successfully managed their emotions.

The correlation coefficient of 0.51 demonstrates that there is a positive and significant relationship between working hours and emotion management. Descriptive data indicate that $41 \%$ of respondents "agreed" with the remote working hours, while the 
same percentage was "indifferent" about it; both groups stated that there are still issues that need to be improved in their organizations.

The coefficient of 0.34 obtained indicates that there is a positive correlation between work and technological support and emotion management. Descriptive data show that $36 \%$ of respondents "agreed" and $35 \%$ were "indifferent" about remote work; only $17 \%$ "disagreed" with it. It can be deduced that most respondents have the necessary tools for the proper management of remote work.

The coefficient of 0.37 obtained indicates that there is a positive correlation between social wellness and emotions management. However, descriptive data indicate that $36 \%$ "disagreed" with the way the organization manages the social wellness of workers during remote work. It is necessary for organizations to pay attention to this dimension, given that social wellness is a non-visible aspect of this work modality.

Based on the results of this research, it is observed that, although not all aspects of remote work are addressed, the majority of the study population, made up of students of Master's in Human Resources at Universidad Nacional Mayor de San Marcos, reported feeling comfortable working remotely and managed their emotions efficiently. Presumably the fact that most of the study participants belong to the millennial generation, who adapt quickly to technological, personal and work-related changes, explains the low level of correlation between the two variables. Nonetheless, public and private organizations need to carefully observe, redesign and better communicate those elements of the working hours, work and technological support and social wellness that were not deemed important. It is a fact that the issues encountered will be unique and different for each organization.

This research leaves open the option for new studies that include other variables such as generational gaps, gender differences, family responsibilities, among others; it serves as a basis for academic study and, above all, for organizations to make better decisions regarding this new form of employment that is probably here to stay.

\section{REFERENCES}

[1] Arbaiza, L. (2016). Cómo elaborar una tesis de grado. Lima, Peru: ESAN Ediciones.

[2] Buitrago, D. (2020). Teletrabajo: una oportunidad en tiempos de crisis. CES Derecho,
11(1), 1-2. Retrieved from https://revistas.ces. edu.co/index.php/derecho/article/view/5620

[3] Cotrufo, T., \& Ureña, J. (2018). El cerebro y las emociones: Sentir, pensar, decidir. Barcelona: Emse Edapp, S.L.

[4] Chávez, L. (February 12, 2021). Trabajo remoto: ¿Por qué los trabajadores están insatisfechos y qué se puede cambiar para el 2021? El Comercio. Retrieved from https://elcomercio. pe/economia/dia-1/trabajo-remoto-por-quelos-trabajadores-no-estan-satisfechos-y-quese-puede-cambiar-para-el-2021-cuarentenaperu-noticia/

[5] Crespo, N. (February 19, 2021): El 21\% de las personas trabajan remotamente de manera regular. FutureLab. Retrieved from https:// futurelab.pe/el-21-de-las-personas-trabajaremotamente-de-manera-regular/

[6] D. S. No. 017-2015-TR. Decreto Supremo que aprueba el Reglamento de la Ley $\mathrm{N}^{\circ}$ 30036, Ley que regula el teletrabajo. Diario Oficial El Peruano (2015). Retrieved from https://busquedas.elperuano.pe/download/url/ decreto-supremo-que-aprueba-el-reglamentode-la-ley-n-30036-decreto-supremo-n-0092015-tr-1307067-3

[7] D. S. No. 008-2020-SA (2020). Decreto Supremo que declara en Emergencia Sanitaria a nivel nacional por el plazo de noventa (90) días calendario y dicta medidas de prevención y control del COVID-19. Diario Oficial EI Peruano (2020). Retrieved from https://busquedas. elperuano.pe/download/url/decreto-supremoque-declara-en-emergencia-sanitaria-a-niveldecreto-supremo-n-008-2020-sa-1863981-2

[8] D. U. No. 026-2020. Decreto de Urgencia que establece diversas medidas excepcionales y temporales para prevenir la propagación del Coronavirus (COVID-19) en el territorio nacional. Diario Oficial El Peruano (2020). Retrieved from https://cdn.www.gob.pe/ uploads/document/file/566447/DU02620201864948-1.pdf

[9] D. U. No. 127-2020. Decreto de Urgencia que establece el otorgamiento de subsidios para la recuperación del empleo formal en el sector privado y establece otras disposiciones. Diario Oficial El Peruano (2020). Retrieved from https://cdn.www.gob.pe/uploads/document/ file/1473551/Decreto\%20de\%20Urgencia $\% 20$ N\%C2\%B0127-2020.pdf.pdf 
[10] Ekman, P. (2013). El Rostro de las Emociones: Qué nos revelan las expresiones faciales. Barcelona: RBA Libros.

[11] Estrategias y Negocio (2021). El futuro del trabajo remoto en el Perú. Estrategias y Negocios. Retrieved from https://eyng.pe/ web/2021/02/11/el-futuro-del-trabajo-remotoen-el-peru/

[12] Eurofound, \& Organización Internacional del Trabajo. (2019). Trabajar en cualquier momento y en cualquier lugar: consecuencias en el ámbito laboral. Oficina de Publicaciones de la Unión Europea, Luxemburgo y Oficina Internacional del Trabajo, Santiago. Retrieved from https://www.ilo.org/wcmsp5/groups/ public/---americas/---ro-lima/---sro-santiago/ documents/publication/wcms_723962.pdf

[13] Gálvez, C. (2017). Inteligencia emocional. En F. Mureira (Ed.), ¿Qué es la inteligencia? (pp. 63-76). Spain: Bubok Publishing S.L.

[14] Gardner, H. (2014). Estructuras de la mente: La teoría de las inteligencias múltiples. Bogotá, Colombia: Fondo de Cultura Económica.

[15] Goleman, D. (1996). La inteligencia Emocional. Por qué es más importante que el cociente intelectual. Buenos Aires, Argentina.

[16] Johnson, M., Saletti-Cuesta, L., \& Tumas, N. (2020). Emociones, preocupaciones y reflexiones frente a la pandemia del COVID-19 en Argentina. Ciência \& Saúde Coletiva, 25, 2447-2456. Retrieved from https://doi. org/10.1590/1413-81232020256.1.10472020

[17] Juárez, J. (2021). El futuro del Trabajo remoto en el Perú. Futurelab. Retrieved from https:// futurelab.pe/el-futuro-del-trabajo-remoto-en-elperu/

[18] Ley No. 30036 (May 15, 2013). Ley que regula el Teletrabajo. Diario Oficial El Peruano (2013). Retrieved from https://busquedas. elperuano.pe/normaslegales/ley-que-regulael-teletrabajo-ley-n-30036-946195-3/

[19] López, J. E. (2020). Flexibilidad, protección del empleo y seguridad social durante la pandemia global del Covid-19. Documentos de Trabajo (IELAT, Instituto Universitario de Investigación en Estudios Latinoamericanos), 134, 1-74. Retrieved from https://dialnet.unirioja.es/ servlet/articulo?codigo $=7393704$

[20] Manucci, M. (2017). Competitividad emocional. Mercado. Retrieved from https://mercado.com. ar/management-marketing/competitividademocional/

[21] Ministerio de Justicia y Derechos Humanos y SERVIR (2016). Guía sobre el sistema administrativo de Gestión de Recursos Humanos en el Sector Público. Guía para asesores jurídicos del Estado. Retrieved from https://www.minjus.gob. pe/wp-content/uploads/2016/07/MINJUSDGDOJ-Gu\%C3\%ADa-sobre-el-SistemaAdministrativo-Servir.pdf

[22] Ministerio de Trabajo y Promoción del Empleo (2019) Manual de preguntas frecuentes laborales. Retrieved from https://cdn.www.gob. pe/uploads/document/file/307150/MANUAL_ DE_PREGUNTAS_FRECUENTES.pdf

[23] Organización Internacional del trabajo OIT (1966). C177 - Convenio sobre el trabajo a domicilio, 1996 (núm. 177). Retrieved from https://www.ilo.org/dyn/normlex/es/f?p=NO RMLEXPUB:12100:0::NO::P12100_ILO_ CODE:C177

[24] Organización Internacional del Trabajo OIT (2020). El teletrabajo durante la pandemia de COVID-19 y después de ella. Guía práctica. Retrieved from https://www.ilo.org/wcmsp5/ groups/public/---ed_protect/---protrav/---travail/ documents/publication/wcms_758007.pdf

[25] Organización Mundial de la Salud (2020). COVID-19: Cronología de la actuación de la OMS. Retrieved from https://www.who.int/es/ news/item/27-04-2020-who-timeline---covid-19

[26] Peralta, A., Bilous, A., Flores, C., \& Bombón, C. (2020). El impacto del teletrabajo y la administración de empresas. Recimundo, $4(1), \quad 326-335$. Retrieved from https:// doi.org/10.26820/recimundo/4.(1). enero.2020.326-335

[27] Rivas, L. A. (2017). Elaboración de Tesis. Estructura y Metodología. Ciudad de México, Mexico: Editorial Trillas.

[28] Salovey, P, \& Mayer, J. D. (1990). Emotional Intelligence. Imagination, Cognition and Personality, 9(3), 185-211. Retrieved from https://doi.org/10.2190/DUGG-P24E-52WK6CDG

[29] Weller, J. (2020). La pandemia del COVID-19 y su efecto en las tendencias de los mercados laborales. Santiago, Chile: CEPAL. Retrieved from https://repositorio.cepal.org/bitstream/ handle/11362/45759/1/S2000387_es.pdf 
